Journal of Engineering and Applied Sciences 14 (6): 1710-1713, 2019

ISSN: 1816-949X

(C) Medwell Journals, 2019

\title{
A Pseudo B-Ideal, Pseudo H-Ideal and a Pseudo Essence of a Pseudo BH-Algebra
}

\author{
Husein Hadi Abbass and Adel Hashem Nouri \\ Department of Mathematics, Faculty of Computer Science and Mathematics, \\ University of Kufa, Kufa, Iraq
}

\begin{abstract}
In this study, we define the notion of a pseudo B-ideal, a pseudo $\mathrm{H}$-ideal and a pseudo essence of a pseudo $\mathrm{BH}$-algebra. Also, we study some properties and relationship between them.

Key words: $\mathrm{BH}$-algebra, ideal of $\mathrm{BH}$-algebra, pseudo $\mathrm{BH}$-algebra, pseudo ideal of a pseudo $\mathrm{BH}$-algebra, pseudo B-ideal, pseudo H-ideal, pseudo essence, pseudo 0-commutative, pseudo G-part, pseudo BCA-part, pseudo closed ideal
\end{abstract}

\section{INTRODUCTION}

Jun et al. (1998) introduced the notion of BH-algebra which is a generalization of $\mathrm{BCH}$-algebra and the notion of ideal of a BH-algebra. Kim and Ahn (2011) introduced the notion of essenceof BH-algebra. Abbass and Dahham (2012) introduced the notion of completely closed ideal of a BH-algebra. By Abbass and Mahdi (2014) introduced the notion of a closed ideal, p-ideal, q-ideal to a BH-algebra and BCA-part. Jun and Kim (2015) introduced the notion of a pseudo BHalgebra.

\section{MATERIALS AND METHODS}

In this sudy, some basic concepts about a $\mathrm{BH}$-algebra, ideal of $\mathrm{BH}$-algebra, essence $\mathrm{BH}$-algebra, 0-commutative BH-algebra, G-part of BH-algebra, BCA part of $\mathrm{BH}$-algebra pseudo, $\mathrm{BH}$-algebra, pseudo subalgebra of a pseudo $\mathrm{BH}$-algebra and pseudo ideal of a pseudo $\mathrm{BH}$-algebra are given.

Definition 1; Jun et al. (1998): A BH-algebra is a nonempty set $\mathfrak{X}$ with constant 0 and a binary operation “*” satisfying the following conditions:

- $\mathrm{x}^{*} \mathrm{x}=0, \forall \mathrm{x} \in \mathrm{X}$

- $\mathrm{x}^{*} 0=\mathrm{x}, \forall \mathrm{x} \in \mathrm{X}$

- $\mathrm{x}^{*} \mathrm{y}=0$ and $\mathrm{y}^{*} \mathrm{x}=0 \Rightarrow \mathrm{x}=\mathrm{y}, \forall \mathrm{x}, \mathrm{y} \in \mathfrak{X}$

Definition 2; Abbass and Dahham (2012): A nonempty subset $\mathrm{S}$ of a $\mathrm{BH}$-algebra $\mathfrak{X}$ is called a subalgebra of $\mathfrak{X}$, if for any $x, y \in S$, we have $x^{*} y \in S$.
Definition 3; Abbass and Dahham (2012): $\mathrm{A}$ BHalgebra $\mathfrak{X}$ is said a 0 -commutative if : $x^{*}\left(0^{*} y\right)=y^{*}\left(0^{*} x\right)$. For all $\mathrm{x}, \mathrm{y}, \mathrm{z} \in \mathfrak{X}$.

Definition 4; Abbass and Mhadi (2014): Let $\mathfrak{X}$ be a $\mathrm{BH}$-algebra. Then the $\operatorname{set} \mathrm{G}(\mathfrak{X})=\left\{\mathrm{x} \in \mathfrak{X}: 0^{*} \mathrm{x}=\mathrm{x}\right\}$ is called G-part.

Definition 5; Abbass and Mahdi (2014): Let $\mathfrak{x}$ be a BH-algebra. Then the set $\mathfrak{X}_{+}=\left\{\mathrm{x} \in \mathfrak{X}: 0^{*} \mathrm{x}=0\right\}$ is called the BCA-part of $\mathfrak{X}$.

Definition 6; Kim and Ahn (2011): Let, $\mathfrak{x}$ be a BH-algebra. For any subsets $\mathrm{G}$ and $\mathrm{H}$ of $\mathfrak{X}$, we define $\mathrm{G}^{*} \mathrm{H}=\left\{\mathrm{x}^{*} \mathrm{y}\right.$ : $\mathrm{x} \in \mathrm{G}$, $\mathrm{yy} \in \mathrm{H}\}$.

Theorem 1; Kim and Ahn (2011): Let a subsets A, B and $\mathrm{E}$ of a $\mathrm{BH}$-algebra, we have:

- $\mathrm{A} \subseteq \mathrm{B} \Rightarrow \mathrm{A}^{*} \mathrm{E} \subseteq \mathrm{B}^{*} \mathrm{E}$ and $\mathrm{E}^{*} \mathrm{~A} \subseteq \mathrm{E}^{*} \mathrm{~B}$

- $(\mathrm{A} \cap \mathrm{B})^{*} \mathrm{E} \subseteq\left(\mathrm{A}^{*} \mathrm{E}\right) \cap\left(\mathrm{B}^{*} \mathrm{E}\right)$

- $\mathrm{E}^{*}(\mathrm{~A} \cap \mathrm{B}) \subseteq\left(\mathrm{E}^{*} \mathrm{~A}\right) \cap\left(\mathrm{E}^{*} \mathrm{~B}\right)$

- $(\mathrm{A} \cup \mathrm{B})^{*} \mathrm{E}=\left(\mathrm{A}^{*} \mathrm{E}\right) \cup\left(\mathrm{B}^{*} \mathrm{E}\right)$

- $\mathrm{E}^{*}(\mathrm{~A} \cup \mathrm{B})=\left(\mathrm{E}^{*} \mathrm{~A}\right) \cup\left(\mathrm{E}^{*} \mathrm{~B}\right)$

Definition 7; Kim and Ahn (2011): If $A$ is a nonempty subset of a $\mathrm{BH}$-algebra $\mathfrak{X}$ satisfies $\mathrm{A}^{*} \mathfrak{X}=\mathrm{A}$, then $\mathrm{A}$ is called essence of $\mathfrak{X}$.

Theorem 2; Kim and Ahn (2011): Let $¥$ be a BH-algebra. Then every a essence of $\mathfrak{X}$ is a subalgebra of $\mathfrak{X}$.

Theorem 3; Kim and Ahn (2011): Let $\mathfrak{X}$ be a BH-algebra. Then, every essence of $\mathfrak{X}$ contains the zero element 0 .

Corresponding Author: Husein Hadi Abbass, Department of Mathematics, Faculty of Computer Science and Mathematics, University of Kufa, Kufa, Iraq 
Definition 8; Jun et al. (1998): Let, $\mathfrak{X}$ be a BH-algebra and $\mathrm{I}(\neq \varnothing) \subseteq \mathfrak{X}$. Then, $\mathrm{I}$ is called an ideal of $\mathfrak{X}$ if it satisfies:

- $0 \in \mathrm{I}$

- If $x^{*} y \in I$ and $y \in I \Rightarrow x \in I$, for all $x \in \mathfrak{X}$

Definition 9: An ideal I of a BCH-algebra is called a closed ideal of $\mathfrak{X}$ if for every $x \in I$, we have $0^{*} x \in I$. We generalize the concept of an ideal to a $\mathrm{BH}$-algebra.

Definition 10: An ideal I of a BH-algebra $\mathfrak{X}$ is called a closed ideal of $\mathfrak{X}$ if: $0^{*} \mathrm{x} \in \mathrm{I}$, for all $\mathrm{x} \in \mathrm{I}$.

Definition 11; Abbass and Dahham (2012): Let $\mathfrak{X}$ be a $\mathrm{BH}$-algebra and I be a subset of $\mathfrak{X}$. Then $\mathrm{I}$ is called a $\mathrm{BH}$-ideal of $\mathfrak{X}$ if it satisfies the following conditions:

- $0 \in \mathrm{I}$

- $x^{*} y \in \mathrm{I}$ and $y \in \mathrm{I}$ imply $\mathrm{x} \in \mathrm{I}$

- $x \in I$ and $y \in \mathfrak{X}$ imply $x^{*} y \in I, I^{*} \mathfrak{X} \subseteq I$

Definition 12; Jun and Kim (2015): A pseudo BH-algebra is a nonempty set $\mathfrak{X}$ with a constant 0 and two binary operations "*" and "\#" satisfying the folloeing condition:

- $\mathrm{x}^{*} \mathrm{x}=\mathrm{x} \# \mathrm{x}=0$

- $\mathrm{x}^{*} 0=\mathrm{x} \# 0=\mathrm{x}$

- $x^{*} y=y \# x=0 \Rightarrow x=y, \forall x, y \in \mathfrak{X}$

Definition 13; Jun and Kim (2015): Let $(\mathfrak{X}, *, \#, 0)$ be a pseudo $\mathrm{BH}$-algebra, then a nonempty subset $\mathrm{S}$ of a pseudo $\mathrm{BH}$-algebra $\mathfrak{X}$ is called a pseudo subalgebra of $\mathfrak{X}$, if for any $x, y \in S$, we have $x^{*} y, x \# y \in S$.

Definition 14; Jun and Kim (2015): Let $\left(\mathfrak{X},{ }^{*}, \#, 0\right)$ be a pseudo BH-algebra, then I is called pseudo ideal of $\mathfrak{X}$, if it satisfies:

- $0 \in \mathrm{I}$

- $x^{*} y, x \# y \in I, y \in I \Rightarrow x \in I, \forall x, y \in \mathfrak{X}$

Definition 15; Jun and Kim (2015): A pseudo ideal I of a pseudo $\mathrm{BH}$-algebra $\mathfrak{X}$ is called a pseudo closed ideal of $\mathfrak{X}$, if for every $\mathrm{x} \in \mathrm{I}$, we have $0^{*} \mathrm{x}, 0 \# \mathrm{x} \in \mathrm{I}$.

\section{RESULTS AND DISCUSSION}

In this study, we define a new types of a pseudo ideals, a pseudo essence subset and a pseudo essence ideal of a pseudo $\mathrm{BH}$-algebra. Also, we study some propostions to other some types of a pseudo ideals of a pseudo BH-algebra.
Definition 1: A pseudo $\mathrm{BH}$-algebra $\mathfrak{X}$ is said a pseudo 0-commutative if:

- $\mathrm{x}^{*}(0 \# \mathrm{H})=\mathrm{y}^{*}(0 \# \mathrm{x})$

- $\quad x \#\left(0^{*} y\right)=y \#\left(0^{*} x\right)$. For all $x, y, z \in \mathfrak{X}$

Example 1: Let $\mathfrak{X}=\{0,1,2\}$ be a set with the following Cayley Table 1 . Then $\mathfrak{X}$ is a pseudo BH-algebra.

Table 1: Pseudo 0-commutative
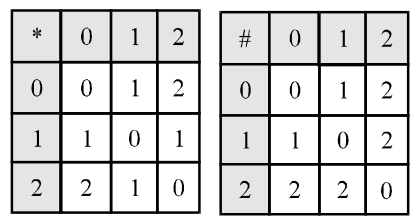

Definition 2: Let be a pseudo BH-algebra. Then thae set $G(\mathbb{X})=\left\{x \in \mathbb{X}: 0^{*} \mathrm{x}=0 \# \mathrm{x}=\mathrm{x}\right\}$ is called a pseudo G-part of .

Example 2: Let $=\{0,1,2,3\}$ be a set with the following Cayley Table 2.

Table 2: Pseudo G-part of

\begin{tabular}{|c|c|c|c|c|c|c|c|}
\hline & 0 & & \begin{tabular}{|l|l|}
2 & 3
\end{tabular} & & & & \\
\hline 0 & 0 & 1 & \begin{tabular}{l|l}
2 & 3 \\
\end{tabular} & 0 & 0 & & \\
\hline & 1 & 0 & \begin{tabular}{l|l}
2 & 3 \\
\end{tabular} & 1 & 1 & 0 & 2 \\
\hline & 2 & 1 & 0 & 2 & 2 & & 0 \\
\hline & & & & & & & \\
\hline
\end{tabular}

Definition 3: Let be a pseudo BH-algebra. Then the set $\mathbb{E}_{+}=\left\{\mathrm{x} \in \mathbb{X}: 0^{*} \mathrm{x}=0 \# \mathrm{x}=0\right\}$ is called a BCA-part of $\mathfrak{X}$.

Example 3: Let $=\{0,1,2,3\}$ be a set with the following Cayley Table 3.

Table 3: Pseudo BCA-part of

\begin{tabular}{|c|c|c|c|c|}
\hline$*$ & 0 & 1 & 2 & 3 \\
\hline 0 & 0 & 0 & 0 & 0 \\
\hline 1 & 1 & 0 & 2 & 3 \\
\hline 2 & 2 & 1 & 0 & 1 \\
\hline 3 & 3 & 3 & 3 & 0
\end{tabular} \begin{tabular}{|c|c|c|c|c|}
\hline$\#$ & 0 & 1 & 2 & 3 \\
\hline 0 & 0 & 0 & 0 & 0 \\
\hline 1 & 1 & 0 & 2 & 3 \\
\hline 2 & 2 & 2 & 0 & 2 \\
\hline 3 & 3 & 3 & 1 & 0 \\
\hline
\end{tabular}

Definition 4: A nonempty subset $I$ of a pseudo $\mathrm{BH}$-algebra $\mathfrak{X}$ is called a pseudo $\mathrm{B}$-ideal of $\mathfrak{X}$ if it stisfies:

- $0 \in \mathrm{I}$

- $x^{*}\left(z \#\left(0^{*} y\right)\right), y \in I$ imply $x^{*} z \in I$

- $x \#(z-(0 \# y)), y \in I$ imply $x \# z \in I$ 
Example 4: Let $=\{0,1,2,3\}$ be a set with the following cayley (Table 4). Then, is a pseudo $\mathrm{BH}$-algebra and let $I=\{0,1\}$ be a subset of then, it is a pseudo B-ideal of .

Table 4: Pseudo B-ideal of

\begin{tabular}{|c|c|c|c|}
\hline$*$ & 0 & 1 & 2 \\
\hline 0 & 0 & 0 & 0 \\
\hline 1 & 1 & 0 & 2 \\
\hline 2 & 2 & 1 & 0 \\
\hline
\end{tabular}$\quad$\begin{tabular}{|l|l|l|l|}
\hline$\#$ & 0 & 1 & 2 \\
\hline 0 & 0 & 0 & 0 \\
\hline 1 & 1 & 0 & 2 \\
\hline 2 & 2 & 2 & 0 \\
\hline
\end{tabular}

Proposition 1: Let, $\mathfrak{X}$ be a pseudo $B H$-algebra such that $\mathfrak{X}=\mathfrak{X}_{+}$, then, every pseudo ideal of $\mathfrak{X}$ is a $\mathrm{B}$-ideal of $\mathfrak{X}$.

Proof: Let, I be a pseudo ideal of $\mathfrak{X}$ and $x^{*}\left(z \#\left(0^{*} y\right)\right)$, yєI. For all $x, y, z \in \mathfrak{X}$. Since, $\mathfrak{X}=\mathfrak{X}_{+} \Rightarrow x^{*}(z \# 0) \in I$. Since, $\mathfrak{X}$ is a pseudo $B H$-algebre $\Rightarrow x^{*} z \in I$. Thus, $x^{*} z \in I$. Similarly, $x \#$ $\left(\mathrm{z}^{*}(0 \# \mathrm{y})\right), \mathrm{y} \in \mathrm{I}$ imply $\mathrm{x} \# \mathrm{z} \in \mathrm{I}$. Hence, $\mathrm{I}$ is a pseudo B-ideal of $\mathfrak{X}$.

Proposition 2: Let $\mathfrak{X}$ be a pseudo $\mathrm{BH}$-algebra. If a pseudo $\mathrm{B}$-ideal of $\mathfrak{X}$ is a pseudo $\mathrm{G}$-part of $\mathfrak{X}$ then, it is a pseudo ideal of $\mathfrak{X}$.

Proof: Let, $\mathfrak{X}$ be a pseudo ideal of $\mathfrak{x}$ andlet $x^{*} y, x \# y \in I$, $y \in I$. For all $x, y, z \in \mathfrak{X}$. Since, $I$ is pseudo G-pert of $\mathfrak{X} \Rightarrow x^{*} 0 \in \mathrm{I} . \Rightarrow x^{*}\left(0 \#\left(0^{*} y\right)\right) \in I$ and $y \in I$. Since, $I$ is a pseudo $\mathrm{B}$-ideal of $\mathfrak{X} \Rightarrow x^{*} O \in I$. Since, $\mathfrak{X}$ is a pseudo $B H$-algebra $\Rightarrow x \in I$. Similarly, $x \# y \in I, y \in I \Rightarrow x \in I$. Hence, $I$ is a pseudo ideal of $\mathfrak{X}$.

Proposition 3: Let $\mathfrak{X}$ be a pseudo $\mathrm{BH}$-algebra such that $y=z \#\left(0^{*} y\right)$ and $y=z^{*}(0 \# y)$, then every pseudo Bideal of $\mathfrak{X}$ is a pseudo ideal of $\mathfrak{X}$.

Proof: Let I be a pseudo B-ideal of $\mathfrak{X}$ and $x^{*}\left(z \#\left(0^{*} y\right)\right)$, $y \in I$. For all $x, y, z \in \mathfrak{X}^{*}$. Since, $y=z \#\left(0^{*} y\right)$ then $x^{*} y, y \in I$ imply $x \in I$. Similarly, $x \#\left(z^{*}(0 \# y)\right)$, yeI imply $x \in I$. Hence, I is a pseudo ideal of $\mathfrak{X}$.

Definition 5: A non empty subset I of a pseudo $\mathrm{BH}$-algebra $\mathfrak{X}$ is called a pseudo $\mathrm{H}$-ideal of $\mathfrak{X}$ if it satisfies:

- $0 \epsilon \mathrm{I}$

- $\left(x^{*} y\right) \#\left(x^{*} z\right) \in \mathrm{I}$ and $y \in \mathrm{I} \Rightarrow \mathrm{x} \in \mathrm{I}$

- $(\mathrm{x} \# \mathrm{y})^{*}(\mathrm{x} \# \mathrm{z}) \in \mathrm{I}$ and $\mathrm{y} \in \mathrm{I} \Rightarrow \mathrm{x} \in \mathrm{I}$. For all $\mathrm{x}, \mathrm{y}, \mathrm{z} \in \mathfrak{X}$

Example 5: Let, $\mathfrak{X}=\{0,1,2,3\}$ be a set with the following Cayley Table 5. Then, $\mathfrak{X}$ is a pseudo BH-algebra and let $\mathrm{I}=\{0,1\}$ be a subset of $\mathfrak{X}$, then it is a pseudo $\mathrm{H}$-ideal of $\mathfrak{X}$.
Table 5: Pseudo H-ideal of $¥$

\begin{tabular}{|c|c|c|c|c|}
\hline$*$ & 0 & 1 & 2 & 3 \\
\hline 0 & 0 & 1 & 2 & 3 \\
\hline 1 & 1 & 0 & 2 & 3 \\
\hline 2 & 2 & 1 & 0 & 2 \\
\hline 3 & 3 & 0 & 0 & 0 \\
\hline
\end{tabular}$\quad$\begin{tabular}{|l|l|l|l|l|}
$\#$ & 0 & 1 & 2 & 3 \\
\hline 0 & 0 & 1 & 2 & 3 \\
\hline 1 & 1 & 0 & 2 & 2 \\
\hline 2 & 2 & 0 & 0 & 1 \\
\hline 3 & 3 & 0 & 0 & 0 \\
\hline
\end{tabular}

Proposition 4: Every pseudo $\mathrm{H}$-ideal of a pseudo $\mathrm{BH}$-algebra is a pseudo ideal of .

Proof: Let, I be a pseudo $H^{*}$-ideal of $\mathfrak{X}$ and let $x^{*} y$, $\mathrm{x} \# \mathrm{y} \in \mathrm{I}$ and $\mathrm{y} \in \mathrm{I}$. For all $\mathrm{x}, \mathrm{y}, \mathrm{z} \in$. Since, $\mathfrak{X}$ is a pseudo $\mathrm{BH}$-algebra $\Rightarrow\left(\mathrm{x}^{*} \mathrm{y}\right) \epsilon \mathrm{I}=\left(\left(\mathrm{x}^{*} \mathrm{y}\right) \# 0\right) \epsilon \mathrm{I}=\left(\mathrm{x}^{*} \mathrm{y}\right) \#\left(\mathrm{x}^{*} \mathrm{x}\right) \epsilon \mathrm{I}$ and $\mathrm{y} \epsilon \mathrm{I}$. Since, $I$ is a pseudo $H$-ideal $\Rightarrow x \in I$. Similarly, $x \# y \in I$ and $y \in I \Rightarrow x \in I$. Hence, $I$ is a pseudo ideal of $\mathfrak{X}$.

Definition 6: Le, $\mathrm{t} \mathfrak{X}$ be a pseudo $\mathrm{BH}$-algebra. For a subsets $\mathrm{A}$ and $\mathrm{B}$ of $\mathfrak{X}$, then $\mathrm{A}^{*} \mathrm{~B}$ and $\mathrm{A} \# \mathrm{~B}$ are defined as follows:

- $\mathrm{A}^{*} \mathrm{~B}=\left\{\mathrm{x}^{*} \mathrm{y}: \mathrm{x} \in \mathrm{A}, \mathrm{y} \in \mathrm{B}\right\}$

- $\mathrm{A} \# \mathrm{~B}=\{\mathrm{x} \# \mathrm{y}: \mathrm{x} \in \mathrm{A}, \mathrm{y} \in \mathrm{B}\}$

Proposition 5: Let, $\mathfrak{X}$ be a pseudo $\mathrm{BH}$-algebra.

- If $0 \epsilon \mathrm{B} \subseteq \mathfrak{X}$. Then $\forall \mathrm{B} \subseteq \mathfrak{X}$, we have $\mathrm{B} \subseteq \mathrm{A}^{*} \mathrm{~B}$ and $\mathrm{B} \subseteq \mathrm{A} \# \mathrm{~B}$

- If $0 \in \mathrm{A} \subseteq \mathfrak{X}$. Then $\forall \mathrm{B} \subseteq \mathfrak{X}$, we have $\mathrm{B} \subseteq \mathrm{A}^{*} \mathrm{~B}$ and $\mathrm{B} \subseteq \mathrm{A} \# \mathrm{~B}$

Proof: Let, $x \in A$, Since, $\mathfrak{X}$ is a pseudo $B H$-algebra, then, $x=x^{*} 0 \in A^{*} B$ and $x=x \# 0 \in A \# B$. Hence, $\left(A \subseteq A^{*} B\right)$ $(A \subseteq A \# B)$. Similarly of (1).

Definition 7: If $\mathrm{A}$ is a nonempty subset of a pseudo $\mathrm{BH}-$ algebra $\mathfrak{X}$ satisfies $\mathrm{A}^{*} \mathfrak{X}=\mathrm{A}$ and $\mathrm{A \#} \mathfrak{X}=\mathrm{A}$, then $\mathrm{A}$ is called a pseudo essence subset of $\mathfrak{X}$. If $\mathrm{A}$ is a pseudo ideal of $\mathfrak{X}$, then it is called a pseudo essence ideal of $\mathfrak{X}$.

Example 6: Let, $\mathfrak{X}=\{0,1,2,3\}$ be a set with the following Cayley Table 6.

Table 6: Pseudo essence of

\begin{tabular}{|c|c|c|c|c|c|c|c|c|c|}
\hline & 0 & 1 & 2 & 3 & \# & 0 & 1 & 2 & \\
\hline 0 & 0 & 0 & 0 & 0 & 0 & 0 & 0 & 0 & 0 \\
\hline 1 & 1 & 0 & 2 & 3 & 1 & 1 & 0 & 2 & 3 \\
\hline 2 & 2 & 1 & 0 & 1 & 2 & 2 & 2 & 0 & 1 \\
\hline 3 & 3 & 3 & 3 & 0 & 3 & 3 & 3 & & 0 \\
\hline
\end{tabular}

Then $\mathfrak{X}$ is a pseudo $\mathrm{BH}$-algebra. Let, $\mathrm{A}=\{0,1\}$, $\mathrm{B}=\{0,2\}$ and $\mathrm{C}=\{0,1,2\}$ then $\mathrm{A}, \mathrm{B}$ and $\mathrm{C}$ are a pseudo 
essence subset of $\mathfrak{X}$. But $\mathrm{D}=\{0,3\}$ is not a pseudo essence subset of $\mathfrak{X}$, since, $3^{*} 2=1 \notin \mathrm{D}$ and $3 \# 2=2 \notin \mathrm{D}$.

Proposition 6: Let, $\mathfrak{X}$ be a pseudo $\mathrm{BH}$-algebra. Then every pseudo essence ideeal of $\mathfrak{X}$ is a pseudo is a pseudo essence subset of $\mathfrak{X}$.

Proof: Let, $A$ be a pseudo ideal of $\mathfrak{X}$ and let $x, y \in A$. Since, $x^{*} y \in A \subseteq A^{*} A \subseteq A^{*} \mathfrak{X}=A$ and $x \# y \in A \subseteq A \# A \subseteq A \# \mathfrak{X}=A$. Hence, $\mathrm{A}$ is a pseudo essence subset of $\mathfrak{X}$.

Remark 1: The converse of prposition (6) may be not true in general as follows in example (1), since, $\mathrm{A}$ is a pseudo essence $1 * 3=0 \in \mathrm{A}$ and $1 \in \mathrm{A}$ but $3 \notin \mathrm{A}$ and $1 \# 3=1 \in \mathrm{A}$ and $1 \in \mathrm{A}$ but $3 \notin \mathrm{A}$.

Proposition 7: Let, $\mathfrak{X}$ be a pseudo $\mathrm{BH}$-algebra. Then, every pseudo essence ideal of $\mathfrak{X}$ is a pseudo closed ideal of $\mathfrak{X}$.

Proof: Let, $\mathrm{A}$ be a pseudo essence ideal of $\mathfrak{X}$, then, $0 \in \mathrm{A}$. Let, $x \in A$, then, $0^{*} x \in A^{*} A \subseteq A^{*} \mathfrak{X}=A$. Thus, $0^{*} x \in A$, similarly, $0 \# x \in A$. Hence, $A$ is a pseudo essence closed of $\mathfrak{x}$.

Definition 8: A nonempty subset $I$ of a pseudo $\mathrm{BH}$-algebra $\mathfrak{X}$. Then, I is called pseudo BH-ideal a of $\mathfrak{X}$ if it satisfies:

- $0 \in \mathrm{I}$

- $x^{*} y, x$ \# $y \in I$ and $y \in I$ imply $x \in I$

- $\quad x \in I$ and $y \in I$ and imply $x^{*} y, x \# y \in I, I^{*} \mathfrak{X}, I \# \mathfrak{X} \subseteq I$. For all $\mathrm{x}, \mathrm{y} \in \mathfrak{X}$
Proposition 8: Let, $\mathfrak{X}$ be a pseudo BH-algebra. Then every a pseudo essence ideal of $\mathfrak{X}$ is a pseudo BH-ideal of $\mathfrak{x}$.

Proof: Let, $A$ be pseudo essence ideal of $\mathfrak{X}$. Since, $\mathrm{A}^{*} \mathfrak{X}=\mathrm{A}$, then $\mathrm{A}^{*} \mathfrak{X} \subseteq \mathrm{A}$ and $\mathrm{A} \# \mathfrak{X}=\mathrm{A}$, then $\mathrm{A} \# \mathfrak{X} \subseteq \mathrm{A}$. Thus, $A$ is a pseudo essence ideal of $\mathfrak{X}$ and $A^{*} \mathfrak{X}$, $\mathrm{A} \# \mathfrak{X} \subseteq \mathrm{A}$. Hence, $\mathrm{A}$ is a pseudo pseudo $\mathrm{BH}$-ideal of $\mathfrak{X}$.

\section{CONCLUSION}

In this study, the notions of pseudo B-ideal, pseudo $\mathrm{H}$-ideal and pseudo essence of a pseudo $\mathrm{BH}$-algebra are introduced. Furthermore, the results are examined in terms of the relationship between pseudo B-idea, pseudo $\mathrm{H}$-ideal and pseudo essence of a pseudo $\mathrm{BH}$-algebra.

\section{REFERENCES}

Abbass, H.H. and A.A. Hamza, 2017. On U-BG-filter of a U-BG-BH-algebra. Appl. Math. Sci., 11: 1297-1305.

Abbass, H.H. and H.A. Dahham, 2012. Some types of fuzzy ideals with respect to an element of a BG-algebra. MSc Thesis, University of Kufa, Kufa, Iraq.

Abbass, H.H. and L.S. Mahdi, 2014. A new class of $\mathrm{BH}$-algebra. MSc. Thesis, University of Kufa, Kufa, Iraq.

Jun, Y.B. and S.S. Kim, 2015. On pseudo BH-algebra. Honam Math. J., 37: 207-219.

Jun, Y.B., E.H. Roh and H.S. Kim, 1998. On BH-algebras. Sci. Math., 1: 347-534.

Kim, E.M. and S.S. Ahn, 2011. An application of complicationness to BH-algebra. J. Korea Soc. Math. Educ., 4: 293-304. 